\title{
Supramalleolar osteotomy combined with lateral ligament reconstruction and talofibular immobilization for varus ankle osteoarthritis with excessive talar tilt angle
}

\author{
Wenqing Qu ${ }^{1,2}$, Dajiang Xin², Shengjie Dong ${ }^{2}$, Wenliang $\mathrm{Li}^{2}$ and Yanping Zheng ${ }^{1,3^{*}}$
}

\begin{abstract}
Background: Although supramalleolar osteotomy is the main joint-preserving method for the treatment of varus ankle osteoarthritis, it tends to be ineffective when ankle osteoarthritis presents in combination with an excessive talar tilt angle. The purpose of this study was to present a new surgical technique, supramalleolar osteotomy combined with lateral ligament reconstruction and talofibular immobilization, for the treatment of varus ankle osteoarthritis with an excessive talus tilt angle and to evaluate the clinical and radiological results.

Methods: From January 2013 to October 2016, a total of 17 patients with 17 cases of varus ankle arthritis with excessive talar tilt angles (larger than $7.3^{\circ}$ ) underwent surgical treatment using our new technique. The American Orthopaedic Foot and Ankle Society (AOFAS) clinical ankle-hindfoot scale and a visual analogue scale (VAS) were used to evaluate ankle function and pain before surgery and at the last follow-up. The medial distal tibial angle (MDTA), anterior distal tibial angle (ADTA), talar tilt angle (TTA), and hindfoot moment arm values (HMAVs) were evaluated on weight-bearing radiographs acquired preoperatively and at the last follow-up.

Results: The AOFAS score improved significantly from $45.8 \pm 2.1$ before surgery to $84.8 \pm 1.8$ after surgery $(p<$ $0.001)$, and the VAS score decreased from $4.9 \pm 0.4$ to $1.1 \pm 0.2(p<0.001)$. The MDTA, TTA, and HMAV changed from $80.9^{\circ} \pm 0.4^{\circ}$ to $90.1^{\circ} \pm 0.4^{\circ}, 11.7^{\circ} \pm 0.6^{\circ}$ to $1.4^{\circ} \pm 0.3^{\circ}$, and $12.6 \mathrm{~mm} \pm 0.8 \mathrm{~mm}$ to $4.2 \mathrm{~mm} \pm 0.6 \mathrm{~mm}$, respectively (each $p<0.001$ ). The ADTA showed no obvious change ( $p=0.370$ ). The staging of 11 cases (65\%) improved. Intramuscular vein thrombosis of the lower limbs occurred in 1 patient 1 week after surgery, and superficial infection occurred in 1 patient.

Conclusions: Supramalleolar osteotomy combined with lateral ligament reconstruction and talofibular immobilization can correct the load of the weight-bearing ankle and effectively improve the ankle function. As the talar tilt angle can be significantly improved after surgery, this technique can be used for the treatment of varus ankle osteoarthritis with an excessive TTA.
\end{abstract}

Keywords: Varus ankle osteoarthritis, Excessive talar tilt angle, Supramalleolar osteotomy, Lateral ligament reconstruction, Talofibular immobilization

\footnotetext{
*Correspondence: 18663827127@163.com

'Qilu Hospital, Shandong University, 107\# Wenhua Xi Road, Jinan 250012,

Shandong, People's Republic of China

${ }^{3}$ Department of Orthopaedics, Qilu Hospital (Qingdao), Shandong University,

758\# Hefei Road, Qingdao 266035, Shandong, People's Republic of China

Full list of author information is available at the end of the article
}

(c) The Author(s). 2019 Open Access This article is distributed under the terms of the Creative Commons Attribution 4.0 International License (http://creativecommons.org/licenses/by/4.0/), which permits unrestricted use, distribution, and reproduction in any medium, provided you give appropriate credit to the original author(s) and the source, provide a link to the Creative Commons license, and indicate if changes were made. The Creative Commons Public Domain Dedication waiver (http://creativecommons.org/publicdomain/zero/1.0/) applies to the data made available in this article, unless otherwise stated. 


\section{Background}

Ankle osteoarthritis (OA) can be manifested as varus, valgus, or neutral alignment, in which varus accounts for approximately $52 \%$ of the cases $[1,2]$. Abnormal alignment often leads to progressive aggravation of ankle osteoarthritis, which gradually progresses to end-stage ankle arthritis. At present, ankle fusion or arthroplasty is the main method for treating end-stage ankle arthritis. However, the former causes loss of ankle motion and the possibility of subsequent adjacent joint degeneration, and the latter may be difficult to perform in patients with preoperative ankle stiffness, high demand for postoperative exercise, osteoporosis, and so on [3, 4]. Therefore, it is of great significance to prevent the progression of moderate ankle arthritis to end-stage arthritis.

A deformation apex of varus ankle arthritis (the center of rotation of angulation, CORA) may peak at the distal tibia, tibia-talus joint, subtalar joint, or at the calcaneus. As the main joint-preserving surgery, supramalleolar osteotomy can adjust the alignment of the tibia and talus and normalize the stress distribution on the ankle joint surface, thus preventing degeneration of the joint; this technique is increasingly widely used [5-8]. Although traditional supramalleolar osteotomy has been reported to be effective [9-13], clinical and radiographic results showed that varus ankle arthritis with an excessive talar tilt angle (TTA) could not be treated effectively. Most authors considered this technique to be ineffective in correcting the TTA if the preoperative TTA was greater than $10^{\circ}[10]$ or $7.3^{\circ}$ [12]. Lee [14] designed a modified supramalleolar osteotomy in response to the problem. However, Ahn et al. [1] found that Lee's osteotomy technique could improve most radiographic parameters satisfactorily, while there was no significant improvement in TTA.

Chronic lateral instability of the ankle (CLIA) can cause varus tilt of the talus and is considered to be the most common cause of varus ankle OA $[2,5]$. In the treatment of varus ankle arthritis, lateral ligament reconstruction is performed with poor improvements to the TTA $[8,12]$. We hypothesized that after lateral ligament reconstruction in supramalleolar osteotomy for the treatment of varus ankle arthritis, immobilization with Kirschner wires for 6 weeks to maintain accurate matching of the talus in the ankle mortise can correct the excessive TTA and achieve a favorable outcome. We investigated the results of this technique for the treatment of varus ankle arthritis with an excessive TTA.

\section{Materials and methods}

\section{Patients}

After Institutional Review Board approval (No. 2018CTECSL-008), we retrospectively reviewed our data from January 2013 to October 2016. The inclusion criteria were as follows: (1) stage 2 or stage 3 adult ankle OA according to Tanaka ankle arthritis staging [12], with clinical symptoms such as ankle swelling, pain, and limited movement; (2) a medial distal tibial angle (MDTA) less than $84^{\circ}$ [15]; (3) a TTA larger than $7.3^{\circ}[12]$; and (4) a follow-up of more than 2 years. Patients with acute or chronic ankle infection, gout or rheumatoid arthritis, neoplastic arthropathy, Charcot arthropathy, neuromuscular dysfunction, lower limb deformity proximal to the ankle, severe ipsilateral subtalar arthritis, and clearly widened ankle mortise or varus deformity of the calcaneus body were excluded.

There were a total of 17 patients, including 13 men and 4 women with a mean age of 56.5 years ( 50 to 65 ). Fifteen patients had a history of severe ankle sprains, 5 had a history of diabetes, and 5 had a history of smoking. There were 2 patients in stage 2, 11 patients in stage 3a, and 4 patients in stage $3 \mathrm{~b}$.

\section{Clinical analysis}

Clinical evaluations were conducted using the American Orthopaedic Foot and Ankle Society (AOFAS) clinical ankle-hindfoot scale [16] and a visual analogue scale (VAS) for pain. The AOFAS score included pain, function, and alignment of the affected limb, with a full score of 100. The VAS score ranged from 0 to 10, depending on the severity of the pain.

\section{Radiological analysis}

Preoperatively and at the last follow-up, weight-bearing radiographs in the anteroposterior view, lateral view, and hindfoot alignment view were applied for the following measurements (Fig. 1): (1) MDTA, the medial angle between the axis of the tibia and the tibial plafond on an anteroposterior radiograph [1]; (2) TTA, the angle between the tibial plafond and talar dome [1]; (3) the anterior distal tibial angle (ADTA), the anterior angle between the axis of the tibia and the tibial plafond on a lateral radiograph [1]; and (4) the hindfoot moment arm values (HMAVs), the distance between the most inferior point on the calcaneus and the tibial line on the hindfoot alignment view [17].

According to their histories of ankle instability, 6 patients underwent anteroposterior and lateral stress radiography. CT scans were performed for 11 patients to investigate the osteophytes and free bodies obstructing the motion of the joints.

\section{Surgical technique}

All surgical procedures were performed by the first author (WQ) and his assistants. Under epidural or general anesthesia, the patient was placed in a supine position with a tourniquet placed on the proximal end of the leg. An anteromedial and an anterolateral incision were made to expose the ankle joint. 


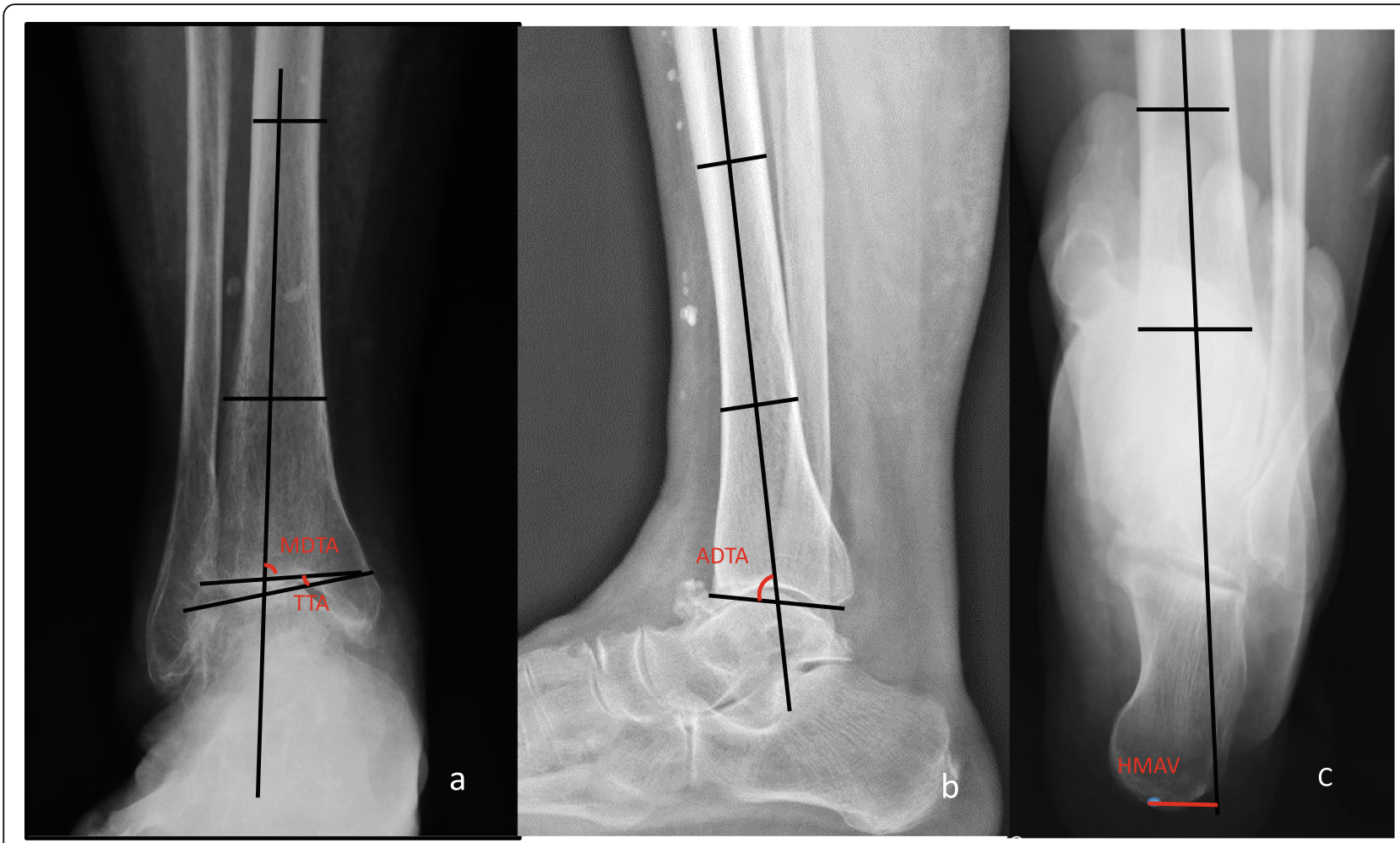

Fig. 1 Female, 62 years old, right varus ankle arthritis, stage 3b. Preoperative weight-bearing anteroposterior radiograph showing a decreased MDTA and increased TTA from neutral (a). Preoperative weight-bearing lateral radiograph showing an increased ADTA from neutral (b). Preoperative weight-bearing hindfoot alignment radiograph showing an increased HMAV from neutral (c)

\section{Joint debridement and distal tibial osteotomy}

The osteophytes were debrided, and the anterior talofibular ligament (ATFL) and calcaneofibular ligament (CFL) were explored. Bone marrow stimulation was performed by cartilage drilling, if necessary [18]. The oblique distal tibial osteotomy procedure was the same as the technique introduced by Lee [14]. A line connecting a point on the medial tibial cortex $5 \mathrm{~cm}$ proximal to the ankle joint and a point on the lateral tibial cortex $5 \mathrm{~mm}$ proximal to the joint was marked by two parallel needles. After the distal tibia was cut using a sharp swing saw, a lamina spreader was inserted for gradual correction of the varus deformity, and the intact lateral tibial cortex was retained as the hinge. After satisfactorily correcting the MDTA and ADTA under fluoroscopy, two wedgeshaped autologous iliac bone grafts were inserted into the defect of the tibia, and an anatomical locking plate was used to complete the fixation. Consistent with Tanaka et al. [12], a fibula osteotomy was performed in 5 patients when intraoperative fluoroscopy showed that the space between the talus and the lateral malleolus was significantly narrowed while the osteotomy site of the tibia was gradually opened.

\section{Reconstruction of the lateral ankle ligaments and talofibular immobilization}

After fixation of the distal tibia, the talus was everted to observe the medial space of the ankle, and the deltoid ligament was released if the medial gutter was narrow. Once the varus and supination deformities of the talus could be corrected passively, and the heel alignment returned to neutral, the modified Brostrom procedure [19] was performed; the lateral ankle ligaments were reinforced by imbrication with the inferior extensor retinaculum using two suture anchors. In all cases, two 2.0$\mathrm{mm}$ Kirschner wires were inserted from the distal fibula into the talus body to maintain the neutral position of the talus. After the drainage tube was placed, the incision was sutured, and the elastic bandage was wrapped to reduce bleeding and prevent DVT. A typical case is shown in Figs. 2 and 3.

\section{Postoperative treatment}

The wound drainage tube was removed approximately $48 \mathrm{~h}$ after surgery. The ankle was kept in a neutral position with a below-knee plaster cast for 6 weeks. Followup was conducted at 6 weeks, 3 months, 6 months, 1 year, and 2 years after surgery. The Kirschner wires for fixation of the lateral malleolus and talus were removed under local anesthesia 6 weeks after surgery, and partial weight-bearing movements were permitted, as tolerated. Full weight-bearing was allowed when complete bone healing was confirmed by radiography approximately 3 to 6 months after surgery. 

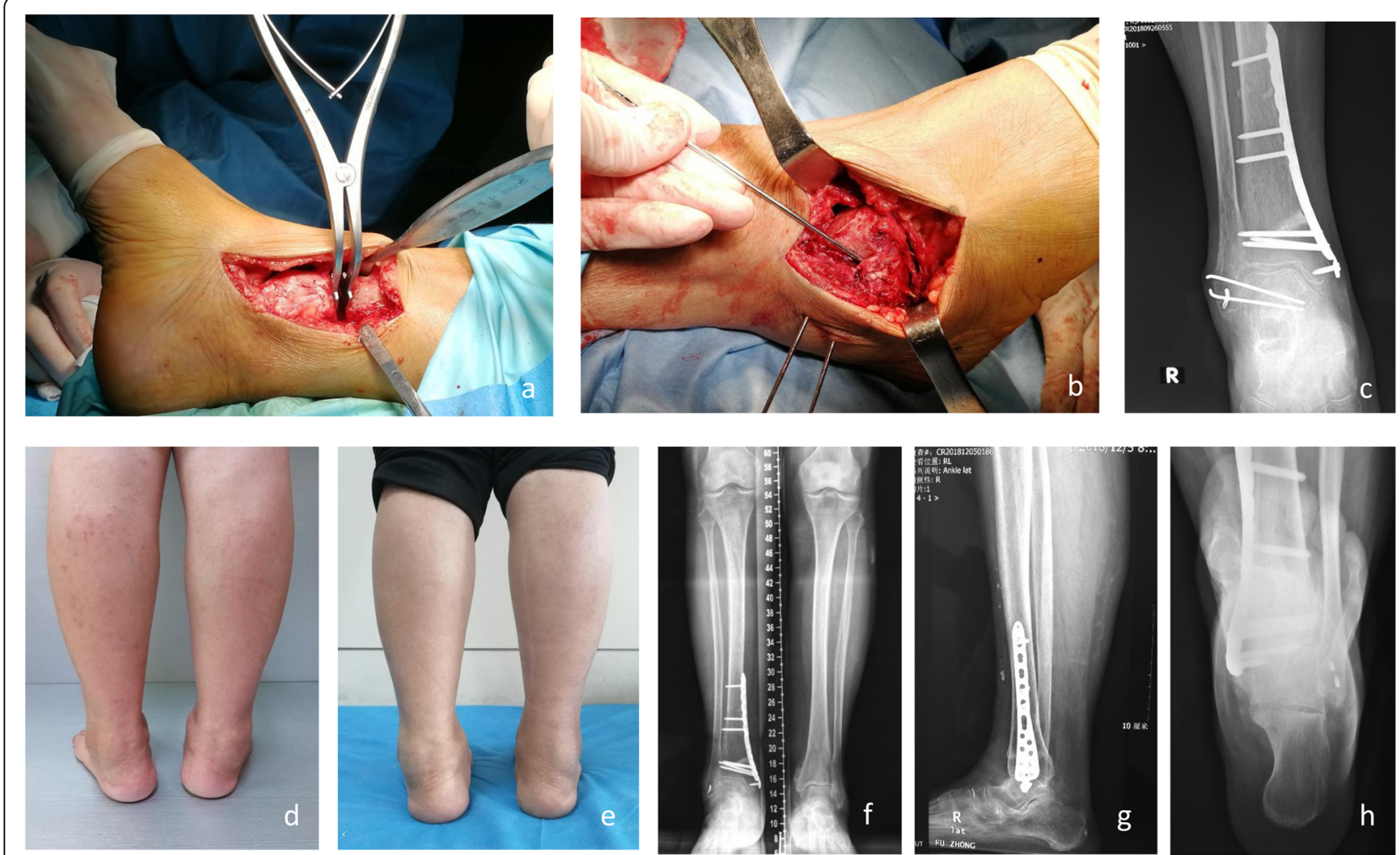

Fig. 2 The treatment process of the same patient shown in Fig. 1. An anteromedial incision (a) was made for joint debridement and supramalleolar osteotomy. An anterolateral incision (b) was made for joint debridement, lateral ankle ligament reconstruction, and Kirschner wire fixation of the tibial talus. Anteroposterior view at 6 weeks postoperatively (c) showed Kirschner wire fixation of the tibial talus and good joint matching. The appearance of the right ankle before (d) and 20 months after surgery (e) showed obvious improvement of the heel alignment. At 20 months after surgery, weight-bearing anteroposterior view (f), lateral view (g), and hindfoot alignment view (h) showed good recovery of the MDTA, ADTA, and TTA. The TTA remained at approximately $0^{\circ}$ and the arthritis stage improved to stage 2

\section{Clinical and radiological analysis}

The AOFAS ankle-hindfoot scale was employed to assess the functional results, and a VAS score was employed to assess pain. Radiological evaluations included measurements of the MDTA, TTA, ADTA, and HMAVs. The matching of the tibia talus and the stage of the ankle joint were observed.

\section{Statistical analysis}

All data were analyzed using SPSS version 21.0 (IBM Corp., Armonk, NY, USA). Continuous variables including the AOFAS score, VAS score, MDTA, ADTA, TTA, and HMAV were presented as the mean \pm standard deviation and were analyzed using paired $t$ tests. Differences were considered statistically significant at $p \leq 0.05$.

\section{Results}

For the group as a whole, the mean follow-up time was 32.2 months (range, 24-40 months). At the last followup, the AOFAS score improved significantly from $45.8 \pm$ 2.1 (range, 35-60 points) preoperatively to $84.8 \pm 1.8$ (range, $66-96$ points) $(p<0.001)$, and the VAS score decreased from $4.9 \pm 0.4$ (range, $2-8$ points) to $1.1 \pm 0.2$ (range, $0-2$ points) $(p<0.001)$.

By radiological evaluations, the MDTA, TTA, and HMAVs changed from $80.9^{\circ} \pm 0.4^{\circ}$ (range, $78-83^{\circ}$ ) to $90.1^{\circ}$ $\pm 0.4^{\circ}\left(\right.$ range, $\left.86-93^{\circ}\right), 11.7^{\circ} \pm 0.6^{\circ}$ (range, $\left.8-17^{\circ}\right)$ to $1.4^{\circ} \pm$ $0.3^{\circ}$ (range, $0-3^{\circ}$ ), and $12.6 \mathrm{~mm} \pm 0.8 \mathrm{~mm}$ (range, $8-18$ $\mathrm{mm}$ ) to $4.2 \mathrm{~mm} \pm 0.6 \mathrm{~mm}$ (range, $0-10 \mathrm{~mm}$ ), respectively (each $p<0.001$ ). The ADTA changed from $82.1^{\circ} \pm 0.3^{\circ}$ (range, $80-84^{\circ}$ ) to $82.5^{\circ} \pm 0.3^{\circ}$ (range, $80-85^{\circ}$ ), and there was no statistical significance $(p=0.370)$ (Table 1$)$.

All patients achieved bone healing in an average of 3.3 months (range, 3-6 months). The arthritis staging improved in 11 cases (65\%); 2 cases improved from stage 2 to stage 1,7 cases from stage 3 a to stage 2, and 2 cases from stage $3 \mathrm{~b}$ to stage $3 \mathrm{a}$. No change was observed in 6 cases, and there were no aggravated cases (Table 2).

Intramuscular vein thrombosis of the lower limbs occurred in 1 patient 1 week after surgery, and superficial infection occurred in 1 patient. No cases of bone nonunion, delayed union, or subsequent ankle fusion were observed during the follow-up. 

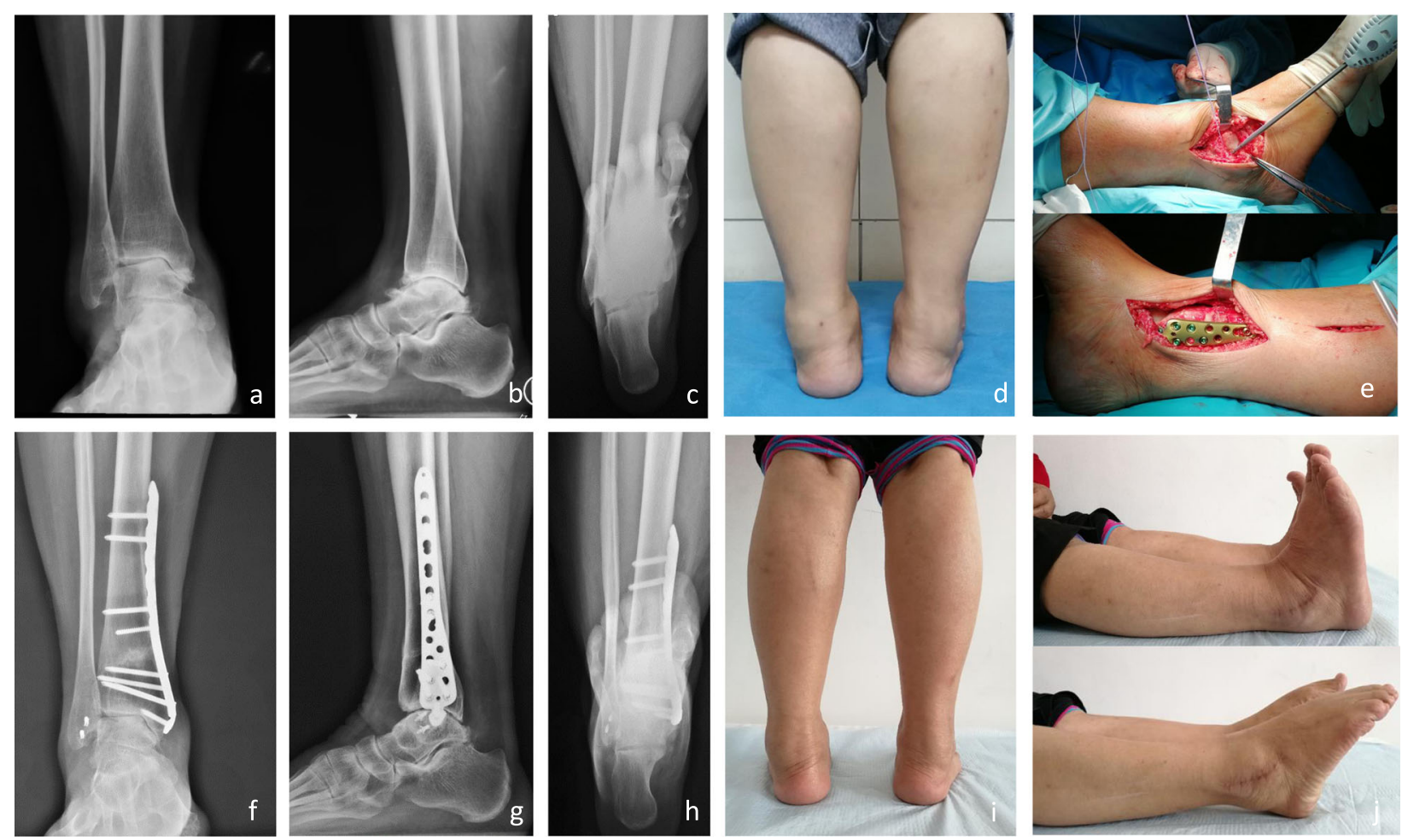

Fig. 3 Male, 55 years old, bilateral ankle arthritis, and the right ankle was treated. Preoperative weight-bearing anteroposterior radiograph (a), lateral radiograph (b), and hindfoot alignment radiograph (c) of the ankle showed a stage 3a ankle arthritis. Photographs obtained on the initial examination of the patient $(\mathbf{d})$. Intraoperative photographs showed lateral ligament reconstruction and medial supramalleolar osteotomy and plate fixation (e). Follow-up radiographs 2 years postoperatively $(\mathbf{f}-\mathbf{h})$ showed good recovery of the MDTA, ADTA, and TTA, and the arthritis stage improved to stage 2 . The good appearance 2 years postoperatively (i). The extension range of motion and the flexion range of motion 2 years postoperatively (j)

\section{Discussion}

The following parameters are significant in the evaluation of varus ankle arthritis deformities. The first parameter is MDTA. The MDTA is applied to assess the varus deformity of the distal tibia, and it varies by region. Hintermann et al. [15] reported the normal MDTA of Europeans ranged from 91 to $93^{\circ}$, while Monji [20] reported the mean value of Japanese was 87.7 to $89.0^{\circ}$. According to a biomechanical study conducted by Stufkens et al. [21], when the lateral malleolus was intact, there was no significant increase in pressure on the lateral half of the tibial talus joint when the axial load was applied after the tibial osteotomy. However, once the lateral malleolus was cut, the pressure on the lateral half of the tibial talus joint increased significantly. This suggests the necessity of considering lateral malleolus osteotomy in supramalleolar osteotomy for patients with lateral malleolus obstruction. A decreased MDTA results in varus deformity above the ankle, high pressure in the medial ankle, accelerated cartilage degeneration, and narrowed

Table 1 Comparison of clinical results and radiological assessment among 17 patients before surgery and at the last follow-up

\begin{tabular}{|c|c|c|c|c|}
\hline Item & Preoperative & Postoperative & Improvement & $p$ value \\
\hline AOFAS score & $45.8 \pm 2.1$ (range $35-60)$ & $84.8 \pm 1.8$ (range 66-96) & 3.0 & $<0.001$ \\
\hline VAS score & $4.9 \pm 0.4$ (range $2-8)$ & $1.1 \pm 0.2$ (range $0-2$ ) & 3.8 & $<0.001$ \\
\hline $\operatorname{MDTA}\left({ }^{\circ}\right)$ & $80.9 \pm 0.4$ (range $78-83)$ & $90.1 \pm 0.4$ (range 86-93) & 9.2 & $<0.001$ \\
\hline $\operatorname{ADTA}\left({ }^{\circ}\right)$ & $82.1 \pm 0.3$ (range $80-84$ ) & $82.5 \pm 0.3$ (range $80-85)$ & 0.4 & 0.370 \\
\hline TTA $\left(^{\circ}\right)$ & $11.7 \pm 0.6$ (range $8-17)$ & $1.4 \pm 0.3$ (range $0-3)$ & 10.3 & $<0.001$ \\
\hline HMAVs (mm) & $12.6 \pm 0.8($ range $8-18)$ & $4.2 \pm 0.6$ (range $0-10$ ) & 8.4 & $<0.001$ \\
\hline
\end{tabular}

Note: All values are expressed as mean \pm standard deviation. Differences were considered statistically significant at $p \leq 0.05$

AOFAS The American Orthopaedic Foot and Ankle Society, VAS visual analogue scale, MDTA medial distal tibial angle, ADTA anterior distal tibial angle, TTA talar tilt angle, HMAVs hindfoot moment arm values 
Table 2 Preoperative and last follow-up ankle OA staging

\begin{tabular}{llllll}
\hline Last & \multicolumn{5}{l}{ Preoperative } \\
\cline { 2 - 6 } follow-up & Stage 1 & Stage 2 & Stage 3a & Stage 3b & Stage 4 \\
\hline Stage 1 & 0 & 2 & 0 & 0 & 0 \\
Stage 2 & 0 & 0 & 7 & 0 & 0 \\
Stage 3a & 0 & 0 & 4 & 2 & 0 \\
Stage 3b & 0 & 0 & 0 & 2 & 0 \\
Stage 4 & 0 & 0 & 0 & 0 & 0 \\
\hline
\end{tabular}

medial joint space [15]. The optimal correction with distal tibia osteotomy is a $2-4^{\circ}$ slight overcorrection of the MDTA [20] or a correction in reference to the normal contralateral limb. With supramalleolar osteotomy, the varus deformity was effectively corrected proximal to the tibial talus joint, leading to restoration of the talar center on the axis of the tibia and dispersion of the stress on the ankle joint surface $[22,23]$. In this study, the mean MDTA improved approximately $10^{\circ}$ after the operation and was considered to be the most important reason for postoperative functional improvement.

The second parameter is TTA. The TTA is applied to assess the varus deformity of the talus on the coronal plane. An excessive TTA is often cited as one of the relative contraindications to supramalleolar osteotomy. Tanaka Y et al. [12] considered a TTA exceeding $7.3^{\circ}$ to be a negative factor for a supramalleolar osteotomy, while Lee WC et al. [10] considered a TTA exceeding $10^{\circ}$ to be a negative factor. A large TTA implies disability of the lateral ligament complex or an erosion of the medial malleolar subchondral bone (widening of the medial mortise space). A proper understanding of large TTAs is helpful in the formulation of targeted surgical procedures, such as arthroplasty $[1,24]$ for intraarticular deformity or lateral ankle ligament reconstruction $[24,25]$ for talar instability. It is meaningful that one of the inclusion criteria of our study was a preoperative TTA larger than $7.3^{\circ}$. The results showed that the TTA decreased significantly after surgery and was maintained effectively during the follow-up; this result was mainly attributed to the release of the deltoid ligament, the reconstruction of the lateral malleolar, and the fixation of the tibia and talus with Kirschner wires to maintain the reduction of the talus in the ankle mortise.

The third parameter is HMAVs [17]. The HMAV is applied to assess the varus deformity of the heel, and the average normal value is $1.6-3.2 \mathrm{~mm}$ [16] inside the tibial axis. The HMAV is more reliable than the tibial heel angle, which is affected by foot rotation [26]. A high HMAV in varus ankle arthritis indicates a varus deformity of the distal tibia, a decrease in the height of the medial half of the talus, or abnormal alignment of the talus and calcaneus. In rare cases, a high HMAV is also associated with varus deformity of the calcaneus body, which sometimes requires lateral sliding calcaneus osteotomy with or without a closing wedge [25]. In addition, when the talus is tilted, the calcaneus may compensate in the opposite direction, resulting in a z-shaped deformity [15]. In this situation, CT scans are recommended to identify the biplane deformity. In this study, the average HMAV changed from $12.6 \mathrm{~mm}$ before surgery to $4.2 \mathrm{~mm}$ after surgery. Although there was significant improvement, the HMAV still did not reach the normal range $(1.6-3.2 \mathrm{~mm})$ [17], which may be related to the coexistence of hidden subtalar joint varus or the uncorrected varus deformity of the calcaneus body.

In moderate to severe ankle osteoarthritis, approximately $60 \%$ of patients present talus varus or valgus, which may be related to talus instability $[15,27]$. The stability of the talus depends on the finely matched bone structure and the healthy soft tissue around the talus, including the medial and lateral collateral ligaments, the ligaments between the talus and calcaneus, and the perimalleolar tendons. Instability of the talus can be caused by abnormal bone structure, such as erosion of the medial malleolus or dysfunction of related ligaments and tendons. In different stages, these abnormalities can exist alone or simultaneously and affect each other. A medial clear space greater than $3 \mathrm{~mm}$ is recommended as a predictor of widened ankle mortise on ankle stress radiographs, and a widened ankle mortise usually implies instability of the talus $[1,28]$.

Lateral instability of the ankle is considered to be an important cause of ankle osteoarthritis. Due to anatomical and biomechanical factors, ankle sprains are more likely to involve the lateral ligaments, which account for approximately $85 \%$ of all ankle sprains; this is consistent with the fact that varus ankle arthritis is more common than valgus ankle arthritis $[1,2,25]$. Relaxation of the anterior talofibular ligament leads to anterior displacement of the talus, which presents as ankle joint subdislocation on anterior stress views in severe cases, while a loose calcaneofibular ligament results in instability of the talus by varus stress, and the combination of the two results in a supination deformity of the talus. Lateral ankle instability can be caused by a single severe injury or multiple minor injuries, and the latter accounts for a higher proportion than the former [29]. The time course of the disease from ankle sprain to ankle joint instability to obvious osteoarthritis is approximately 20 years [30,31], which suggests that the early treatment of lateral ankle ligament injuries should be emphasized to prevent chronic ankle joint instability and osteoarthritis. In this group, 15 patients had one or more previous obvious ankle sprains, which were believed to be related to the development of arthritis.

The correction of an excessive TTA is a difficult problem in ankle salvage surgery. Talar tilt is often accompanied by erosion of the media malleolus and widening 
of the medial gutter. Myerson and Zide recommend plafondplasty (intra-articular osteotomy) to narrow the mortise and reduce the TTA [32]. However, Lee believed that the procedure only corrected the medial half dome and may cause new intra-articular deformities; thus, they designed the distal tibial oblique osteotomy to correct the tilt of the entire tibial dome and to narrow the ankle mortise without damaging the joint surface and distal tibiofibular syndesmosis [14]. However, other authors considered that Lee's technique was effective in correcting the width of ankle mortise but not effective when the TTA was too large [1].

The need for reconstruction of the lateral malleolar ligaments after distal tibial osteotomy is controversial. Lee et al. [25] and Mann et al. [24] believed that reconstruction was an essential adjunct to bony osteotomy and that the talus could be accurately stabilized in the ankle mortise. However, other authors believed that the abnormal stress within the ankle joint would be distributed after the realignment of the lower limbs, and ligament reconstruction was unnecessary $[8,9,12]$. We believe that if the treatment of ankle arthritis with a significantly increased TTA is not combined with reconstruction of the lateral ligament, the supination deformity of the talus would still exist, and the stress on the medial side of the ankle would remain too high while weight-bearing. The lateral ligament reconstruction technique (the modified Brostrom operation) used in this study is the gold standard for the treatment of CLIA; however, Lee et al. [25] showed that this technique was not effective when the TTA was too large. We considered this result to be due to the redisability of the ligaments postoperatively. To avoid the re-relaxation of the reconstructed ligaments, we fixed the fibula and talus with two 2.0-mm Kirschner wires, immobilized the ankle joint with a short leg brace after surgery, and removed the Kirschner wires 6 weeks postoperatively. The mechanism is similar to the postoperative external fixator fixation mechanism after osteotomy introduced by Zhao et al. [33] but is more simple and minimally invasive.

Some limitations of the present study must be acknowledged. A small number of cases were included in our study, and there was no control group to confirm the positive effect of the 6-week postoperative tibia-talus fixation by Kirschner wires. In addition, the mean follow-up time was 32.2 months, but the range was large (24-40 months), which may have an impact on the efficacy results. Moreover, this study has not successfully analyzed the individual factors (high TTA, severity of arthritis preoperatively, age, etc.) that might be perhaps predictive of success or failure as a result of the sample size. Large-scale and long-term follow-up studies are needed to provide more convincing clinical data for the application of this technique.

\section{Conclusion}

Supramalleolar osteotomy combined with lateral ligament reconstruction and talofibular immobilization can effectively improve the load of the ankle in the treatment of varus ankle arthritis. As the talar tilt angle can be significantly improved and effectively maintained, this technique could be an alternative method for the treatment of varus ankle arthritis with an excessive TTA.

\section{Abbreviations \\ ADTA: Anterior distal tibial angle; AOFAS: American Orthopaedic Foot and Ankle Society; ATFL: Anterior talofibular ligament; CFL: Calcaneofibular ligament; CLIA: Chronic lateral instability of the ankle; CORA: Center of rotation of angulation; HMAVs: Hindfoot moment arm values; MDTA: Medial distal tibial angle; OA: Osteoarthritis; TTA: Talar tilt angle; VAS: Visual analogue} scale

\section{Acknowledgements}

We would like to thank Editage [www.editage.cn] for the English language editing.

\section{Authors' contributions}

WQQ and YPZ performed the study design, analyzed the results, and contributed to the manuscript. DJX made some meaningful suggestions. SJD and WLL contributed to the collection of the cases. All authors reviewed and approved the final submitted version.

\section{Funding}

This study was supported by the Key Research and Development Plan of Yantai Science and Technology Bureau (2016WS033), Key Research and Development Projects of Shandong (2016GSF201112), and Technology Development Plan of Shandong Medical Health Bureau (2016WS0696).

Availability of data and materials

All data used and analyzed during this study are available from the corresponding author on reasonable request.

Ethics approval and consent to participate

The study was approved by Institutional Review Board of Yantaishan Hospital, (No. 2018CTEC-SL-008). Written informed consent was obtained from all patients.

\section{Consent for publication}

Not applicable.

\section{Competing interests}

The authors declare that they have no competing interests.

\section{Author details}

${ }^{1}$ Qilu Hospital, Shandong University, 107\# Wenhua Xi Road, Jinan 250012, Shandong, People's Republic of China. ${ }^{2}$ Department of Orthopaedics, Yantaishan Hospital, 91\# Jiefang Road, Zhifu District, Yantai 264001, Shandong, People's Republic of China. ${ }^{3}$ Department of Orthopaedics, Qilu Hospital (Qingdao), Shandong University, 758\# Hefei Road, Qingdao 266035, Shandong, People's Republic of China.

Received: 3 May 2019 Accepted: 5 November 2019

Published online: 28 November 2019

References

1. Ahn TK, Yi Y, Cho JH, Lee WC. A cohort study of patients undergoing distal tibial osteotomy without fibular osteotomy for medial ankle arthritis with mortise widening. J Bone Joint Surg Am. 2015;97:381-8.

2. Valderrabano $V$, Hintermann B, Horisberger M, Fung TS. Ligamentous posttraumatic ankle osteoarthritis. Am J Sports Med. 2006;34:612-20.

3. Robert F, Coleman SC, Shay T, Brodsky JW. Comparison of gait after total ankle arthroplasty and ankle arthrodesis. Foot Ankle Int. 2013;34:1340-8.

4. Swati C, Hossein R, Mathieu A, Aminian K, Crevoisier X. Outcome of unilateral ankle arthrodesis and total ankle replacement in terms of a cohort 
study of patients undergoing distal tibial osteotomy without fibular osteotomy for medial ankle arthritis with mortise widening bilateral gait mechanics. J Orthop Res. 2014;32:377-84.

5. Hintermann Z, Lukas RR, Barg A. The use of supramalleolar osteotomies in posttraumatic deformity and arthritis of the ankle. JBJS Essent Surg Tech. 2017;7:e29.

6. Watanabe K, Teramoto A, Kobayashi T, Sakakibara Y, Shoji H, Okimura S, et al. Modified distal tibial oblique osteotomy for osteoarthritis of the ankle: operative procedure and preliminary results. J Orthop Sci. 2019;24:306-11.

7. Barg A, Pagenstert Gl, Horisberger M, Paul J, Gloyer M, Henninger HB, et al. Supramalleolar osteotomies for degenerative joint disease of the ankle joint: indication, technique and results. Int Orthop. 2013;37:1683-95.

8. Hayato K, Yasunori K, Yoji S. Treatment of varus ankle osteoarthritis and instability with a novel mortise-plasty osteotomy procedure. J Foot Ankle Surg. 2016:55:60-7.

9. Takakura Y, Tanaka Y, Kumai T, Tamai S. Low tibial osteotomy for osteoarthritis of the ankle. Results of a new operation in 18 patients. J Bone Joint Surg Br. 1995;77:50-4

10. Lee WC, Moon JS, Lee K, Byun WJ, Lee SH. Indications for supramalleolar osteotomy in patients with ankle osteoarthritis and varus deformity. J Bone Joint Surg Am. 2011:93:1243-8.

11. Stamatis ED, Cooper PS, Myerson MS. Supramalleolar osteotomy for the treatment of distal tibial angular deformities and arthritis of the ankle joint. Foot Ankle Int. 2003;24:754-64

12. Tanaka Y, Takakura Y, Hayashi K, Taniguchi A, Kumai T, Sugimoto K. Low tibial osteotomy for varus-type osteoarthritis of the ankle. J Bone Joint Surg Br. 2006;88:909-13.

13. Pagenstert Gl, Hintermann B, Barg A, Leumann A, Valderrabano V. Realignment surgery as alternative treatment of varus and valgus ankle osteoarthritis. Clin Orthop Relat Res. 2007:462:156-68.

14. Lee WC. Extraarticular supramalleolar osteotomy for managing varus ankle osteoarthritis, alternatives for osteotomy: how and why? Foot Ankle Clin. 2016;21:27-35

15. Hintermann B, Knupp M, Barg A. Joint-preserving surgery of asymmetric ankle osteoarthritis with peritalar instability. Foot Ankle Clin. 2013;18:503-16.

16. Kitaoka HB, Alexander IJ, Adelaar RS, Nunley JA, Myerson MS, Sanders M. Clinical rating systems for the ankle-hindfoot, midfoot, hallux, and lesser toes. Foot Ankle Int. 1994:15:349-53.

17. Saltzman CL, El-Khoury GY. The hindfoot alignment view. Foot Ankle Int 1995;16:572-6.

18. Kim YS, Park EH, Koh YG, Lee JW. Supramalleolar osteotomy with bone marrow stimulation for varus ankle osteoarthritis. Am J Sports Med. 2014:42:1558-66.

19. Gould N, Seligson D, Gassman J. Early and late repair of lateral ligament of the ankle. Foot Ankle. 1980;1:84-9.

20. Monji J. Roentgenological measurement of the shape of the osteoarthritic ankle. J Jpn Orthop Assoc. 1980;54:791-802 (in Japanese).

21. Stufkens SA, van Bergen CJ, Blankevoort L, van Dijk CN, Hintermann B, Knupp M. The role of the fibula in varus and valgus deformity of the tibia: a biomechanical study. J Bone Joint Surg Br. 2011;93:1232-9.

22. Tanaka Y. The concept of ankle joint preserving surgery: why does supramalleolar osteotomy work and how to decide when to do an osteotomy or joint replacement. Foot Ankle Clin. 2012;17:545-53.

23. Knupp M, Stufkens SA, Bolliger L, Barg A, Hintermann B. Classification and treatment of supramalleolar deformities. Foot Ankle Int. 2011;32:1023-31.

24. Mann HA, Filippi J, Myerson MS. Intra-articular opening medial tibial wedge osteotomy (plafond-plasty) for the treatment of intra-articular varus ankle arthritis and instability. Foot Ankle Int. 2012:33:255-61.

25. Lee HS, Wapner KL, Park SS, Kim JS, Lee DH, Sohn DW. Ligament reconstruction and calcaneal osteotomy for osteoarthritis of the ankle. Foot Ankle Int. 2009;30:475-80.

26. Reilingh ML, Beimers L, Tuijthof GJ, Stufkens SA, Maas M, van Dijk CN. Measuring hindfoot alignment radiographically: the long axial view is more reliable than the hindfoot alignment view. Skeletal Radiol. 2010;39:1103-8.

27. Lee WC, Moon JS, Lee HS, Lee K. Alignment of ankle and hindfoot in early stage ankle osteoarthritis. Foot Ankle Int. 2011;32:693-9.

28. Park SS, Kubiak EN, Egol KA, Kummer F, Koval KJ. Stress radiographs after ankle fracture: the effect of ankle position and deltoid ligament status on medial clear space measurements. J Orthop Trauma. 2006;20:11-8.

29. Knupp M, Lang TH, Zwicky L, Lötscher P, Hintermann B. Chronic ankle instability (medial and lateral). Clin Sports Med. 2015;34:679-88.
30. Horisberger M, Valderrabano V, Hintermann B. Posttraumatic ankle osteoarthritis after ankle-related fractures. J Orthop Trauma. 2009:23:60-7.

31. Palmer-Green DS, Batt ME, Scammell BE. Simple advice for a simple ankle sprain? The not so benign ankle injury. Osteoarthritis Cartilage. 2016;24:947-8.

32. Myerson MS, Zide JR. Management of varus ankle osteoarthritis with jointpreserving osteotomy. Foot Ankle Clin. 2013;18:471-80.

33. Zhao HM, Liang XJ, Li Y, Ning N, Lu J. Supramalleolar osteotomy with distraction arthroplasty in treatment of varus ankle osteoarthritis with large talar tilt angle: a case report and literature review. J Foot Ankle Surg. 2017; 56:1125-8.

\section{Publisher's Note}

Springer Nature remains neutral with regard to jurisdictional claims in published maps and institutional affiliations.
Ready to submit your research? Choose BMC and benefit from:

- fast, convenient online submission

- thorough peer review by experienced researchers in your field

- rapid publication on acceptance

- support for research data, including large and complex data types

- gold Open Access which fosters wider collaboration and increased citations

- maximum visibility for your research: over $100 \mathrm{M}$ website views per year

At $\mathrm{BMC}$, research is always in progress.

Learn more biomedcentral.com/submissions 